Reprod. Nutr. Dévelop., 1981, 21 (3), 399-407.

\title{
Variations saisonnières de la qualité du sperme chez le bélier Ile-de-France. II. Fécondance : relation avec les critères qualitatifs observés in vitro
}

\author{
par G. COLAS \\ avec la collaboration technique de Y. GUÉRIN
}

Station de Physiologie de la Reproduction, I.N.R.A., Nouzilly, 37380 Monnoie, France.

Summary. Seasonal variations of semen quality in adult lle-de-France rams. II. Fertilizing ability and its relation to qualifative criteria in vitro.

Six adult lle-de-France rams of different origins, registered in a Flock book, were used in Al for three consecutive years on the same flock (IF) in March-April and in September. The animals (males and females) were always kept in a sheep-fold (natural daylight conditions). They received the same feed throughout the whole experiment.

Using the unselected ejaculate(s) of each ram (1 dose diluted sperm/female), artificial inseminations were carried out after oestrus synchronization (FGA + PMSG).

Massal motility, percentage of nigrosin-eosin-stained spermatozoa and percentage of morphologically abnormal sperm were determined (150 cells/smear) on each ejaculate (or pooled ejaculates of each ram) used for insemination.

1. - In the spring, sperm fertility was strongly diminished (47.1 vs 68.4 p. 100 LR; $P<0.01$, respectively, in spring and autumn), and the differences between the rams were significant (31.7 to 70.3 p. $100 ; 0.01<\mathrm{P}<0.02$ in spring vs 60.0 to $80.0 ; \mathrm{P}>0.05$ in autumn).

2. - Fertilizing ability was related neither to massal motility nor to the proportion of stained cells, although, on the whole, the latter was somewhat higher in spring than in autumn.

In the spring, there was a close relationship between the fertility of the ejaculate and its proportion of abnormalities $(r=-0.83 ; P<0.01)$.

Fertility was also related to the percentage of abnormal heads $(r=-0.59 ; P<0.05)$. In autumn, when the proportion of total abnormalities was much lower and almost exclusively made up of slight tail damages, this relationship disappeared. Morphological examination of the semen is thus a good fertility test.

These results show the importance of the ram in successful breeding in an anoestrous period. Morphological test in the spring must be used to detect those males which are most affected by increasing daylight since, at that time (March-April), a ram maintains nearly the same morphological profile from one year to the next (Colas, 1980).

\section{Introduction.}

Dans une première étude (Colas, 1980), nous avons montré que la qualité des spermatozoïdes de bélier et en particulier leur morphologie subissent d'assez fortes 
variations saisonnières. Dans le présent travail, nous nous sommes demandé si le pouvoir fécondant de la semence évolue lui aussi au cours de l'année et quelle relation existe entre nos mesures effectuées in vitro et la fertilité.

Si l'on a quelques éléments de réponse en ce qui concerne la première question, (Salamon et Robinson, 1962 ; Fowler, 1965 ; Colas et Brice, 1976), on ne possède en revanche aucune information précise à propos de la seconde. Encore, faut-il indiquer que les résultats publiés par ces auteurs sont obtenus la plupart du temps à partir de femelles en œstrus naturel. Ce manque de données explique qu'aujourd'hui encore, la sélection des éjoculats soit conduite de manière très empirique chez les ovins.

Nous avons donc repris cetle étude en nous plaçant dans les conditions pratiques de l'insémination artificielle en France, c'est-à-dire en utilisant des brebis dont l'ovulation a été induite par un traitement hormonal.

\section{Matériel et méthodes.}

1. Animaux expérimentaux. - Six béliers adultes ( 3 de 3 ans, 3 de 4 ans), inscrits au Livre généalogique de la race lle-de-France et n'ayant entre eux aucun lien de parenté, ont été ułilisés en insémination artificielle (IA) pendant trois années consécutives (1977 à 1979). Leurs conditions d'entretien (bergerie, alimentation) ont été précisées dans l'étude antérieure consacrée à l'examen in vitro de la semence de 5 de ces animoux (Colas, 1980).

Les brebis, toutes de même race, étaient âgées d'au moins un an au moment des essais et provenaient du même troupeau. Leur régime alimentaire a été maintenu constant tout au long de la période expérimentale.

2. Contrôle du sperme. - Sur chaque éjaculat (ou groupe d'éjaculats du même bélier) utilisé in vivo (IA) nous avons procédé aux examens suivants :

- motilité massale (1977 à 1979),

- pourcentage de spermatozoïdes colorés à l'éosine-nigrosine (1978 et 1979),

- pourcentage de spermatozoïdes anormaux (1978 et 1979).

Les contrôles de la motilité et du pourcentage d'anomalies se sont déroulés dans des conditions identiques à celles que nous avons décrites précédemment (Colas, 1980). Les pourcentages de cellules colorées ou anormales étaient déterminés à partir des mêmes frottis (150 cellules/lame).

Toutes les mesures ont été réalisées par la même personne.

3. Essais de fécondance. - Chaque année (1977 à 1979), aux mêmes époques (mars ou avril et septembre), des essais de fécondance ont éfé réalisés à partir des éjaculats (ou groupe d'éjaculats) non triés de chacun des six béliers. Ces collectes étaient différentes de celles déjà effectuées pour l'étude de la morphologie cellulaire (Colas, 1980).

Les brebis ont reçu de l'acétate de fluorogestone par voie vaginale $(30 \mathrm{mg}$ au printemps, $40 \mathrm{mg}$ en automne) et de la PMSG en injection IM (600 UI au printemps, $400 \mathrm{UI}$ en automne). $55 \pm 1 \mathrm{~h}$ après le retrait de l'éponge, elles ont été inséminées avec une seule dose de semence conservée quelques heures à $+15^{\circ} \mathrm{C}$ dans du lait 
écrémé additionné d'antibiotiques et de sulfamides $\left(500 \times 10^{6}\right.$ spz/q en 1977 ; $400 \times 10^{6} \mathrm{spz} /$ 果 en 1978 et 1979) (Colas, 1975, 1979). Ces interventions ont été réalisées sur des femelles dont l'intervalle part-insémination était d'au moins 80 jours.

4. Expression ef analyse des résultats. - La fertilité est exprimée en taux de misesbas, que nous avons comparés à l'aide du test $\chi^{2}$ de Pearson.

L'étude des pourcentages de spermatozoïdes colorés ou anormaux (土 écarttype) a été effectuée par analyse de variance après transformation angulaire des données de base.

Dans le cas des anomalies totales, nous n'avons procédé au calcul du coefficient de corrélation (après transformation angulaire des données) que si l'étude graphique révélait l'existence d'une relation.

Les motilités moyennes sont exprimées sans écart-type, la distribution des valeurs individuelles n'étant pas normale.

\section{Résultats.}

1. Variations saisonnières de la fécondance.

A. Ełude globale. - La fécondance moyenne des six béliers est chaque année plus faible au printemps qu'en automne (tabl. 1). Toutefois, cette différence n'est significative qu'au cours des 2 dernières années $(P<0,05)$ et sur l'ensemble de la période expérimentale $(P<0,01)$.

\section{TABLEAU 1}

Influence de la saison sur la fécondance (p. 100 M.B.)

des béliers lle-de-France ( $n=6$, éjaculats non triés)

\begin{tabular}{ccc}
\hline Année & $\begin{array}{c}\text { Printemps } \\
\text { (mars-arril) }\end{array}$ & $\begin{array}{c}\text { Automne } \\
\text { (septembre) }\end{array}$ \\
\hline $1977 \ldots \ldots \ldots \ldots$ & $45,7_{(35)}^{\mathrm{a}}$ & $64,0_{(78)}^{\mathrm{a}}$ \\
$1978 \ldots \ldots \ldots \ldots$ & $49,3_{(69)}^{\mathrm{b}}$ & $74,4_{(86)}^{\mathrm{c}}$ \\
$1979 \ldots \ldots \ldots$ & $45,8_{(72)}^{\mathrm{d}} \ldots \ldots$ & $66,7_{(99)}^{\mathrm{e}}$ \\
\hline TOTAL..... & $47,1_{(176)}^{\mathrm{f}}$ & $68,4_{(263)}^{\mathrm{g}}$ \\
\hline
\end{tabular}

( ) : Brebis inséminées $55 \pm 1 \mathrm{~h}$ après le retrait de l'éponge ef l'injection de PMSG.

Pour une même année, les pourcentages affectés d'une même lettre ne sont pas sfatistiquement différents au seuil 5 p. 100.

On remarque également que les taux de mise-bas semblent moins variables au printemps qu'en automne : écart entre les extrêmes 3,6 contre 10,3 points.

B. Différences individuelles. - Pour un même bélier, à l'intérieur d'une même saison, les taux de fertilité n'étant pas significativement différents entre années, nous les avons regroupés en une valeur moyenne par saison et par mâle (tabl. 2). 
TABLEAU 2

Fécondance individuelle des béliers lle-de-France $(\mathrm{n}=6)$, au printemps (mars ou avril) ef en automne (septembre) (moyenne de 3 années)

\begin{tabular}{|c|c|c|c|}
\hline \multirow{2}{*}{ Bélier } & \multicolumn{2}{|c|}{ Fertilité (p. 100 M.B.) } & \multirow{2}{*}{ Signification } \\
\hline & Printemps & Automne & \\
\hline 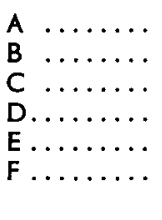 & $\begin{array}{c}41,7_{(24)} \\
70,3_{(37)} \\
53,1_{(32)} \\
33,3_{(21)} \\
31,7_{(41)} \\
\left.47,6_{(21)}\right) \\
0,01<P<0,02\end{array}$ & $\begin{array}{c}61,7_{(34)} \\
60,0_{(30)} \\
66,7_{(66)} \\
76,8_{(56)} \\
63,8_{(47)} \\
80,0_{(30)} \\
N S\end{array}$ & $\begin{array}{c}N S \\
N S \\
N S \\
P<0,01 \\
P<0,01 \\
P<0,05\end{array}$ \\
\hline
\end{tabular}

( ) : Brebis inséminées à partir d'éjaculats non triés.

Au printemps, la fécondance évolue de façon très marquée selon les béliers (31,7 à 70,3 p. 100, 0,01 $<\mathrm{P}<0,02)$. En automne, les écarts sont moins importants $(60,0$ à 80,0 p. 100$)$ ef non significatifs.

2. Relation entre les critères qualitatifs des éjaculats ef leur fécondance.

Sur la figure 1, nous avons représenté la fécondance de chaque éjaculat en fonction des paramètres qualitatifs étudiés. Pour les deux premiers paramètres (motilité, cellules colorées), les résultats de printemps et d'automne ont été réunis sur le même graphique. Pour les spermatozoïdes anormaux, par contre, nous avons préféré dissocier les données des deux saisons, les malformations du flagelle étant beaucoup plus marquées ef plus graves au printemps qu'en automne.
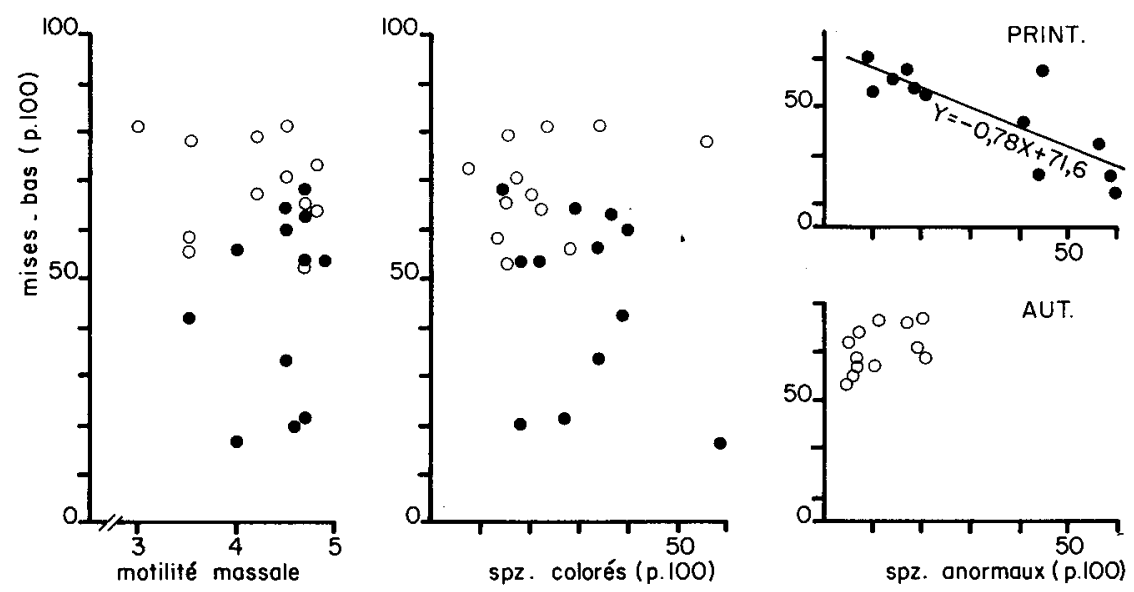

FIG. 1. - Relation entre les critères qualitaifs des éjaculats de bélier et leur fécondance au printemps (๑) et à l'automne (o). 
A. Motilité massale. - Sur l'ensemble des trois années et à l'intérieur de chaque saison, il n'existe aucune relation entre la motilité massale du sperme et sa fécondance (fig. 1). La motilité moyenne des éjaculats utilisés au printemps et en automne varie d'ailleurs très peu d'une année sur l'autre (respectivement 4,4 vs 4,6 en $1977,4,5$ vs 4,2 en $1978,4,0$ vs 4,0 en 1979).

B. Spermatozoìdes colorés. - Dans l'ensemble, la proportion de spermatozoïdes colorés des éjaculats est plus élevée en mars-avril qu'en septembre, mais la différence n'est significative que la première année : $30,1 \pm 1,6$ vs $18,5 \pm 1,3$ p. $100, P<0,05$ et $29,3 \pm 3,0$ vs $23,7 \pm 3,2$ p. $100, P>0,05$ respectivement en 1978 et 1979 . Toutefois, lorsqu'on considère les éjaculats un à un, cette proportion n'est reliée en aucune saison au pouvoir fécondant (fig. 1).

\section{Spermatozoïdes anormaux.}

- Anomalies totales. Au printemps (fig. 1), la fécondance des éjaculats est liée de façon inverse à leur pourcentage d'anomalies morphologiques. La droiłe de régression de $Y$ ( $p .100 \mathrm{MB}$ ) en $X$ (p. 100 anomalies) a pour equation :

$$
Y=-0,78 x+71,6(r=-0,83, P<0,01) \text {. }
$$

Ainsi, à toute élévation du pourcentage d'anomalies de 10 points correspond une baisse de fertilité d'environ 8 points.

En automne, au contraire, le diagramme ne révèle plus aucune relation entre les deux variables mais à cefte saison, la proportion d'anomalies autres que celles du flagelle varie de 0 à 4,7 p. 100 selon les béliers. On constate aussi que l'ensemble des malformations varie dans des limites plus faibles en automne (4,7 à 20,0 p. 100) qu'au printemps (9,3 à 59,3 p. 100).

- Anomalies de chaque catégorie. Le calcul des corrélations entre les différentes catégories d'anomalies et la fécondance n'a été possible qu'au printemps (pour la raison que nous venons d'évoquer) et seulement pour les malformations du flagelle et de la tête qui sont les deux formes que nous avons rencontrées en permanence dans les 12 éjaculats.

Les valeurs obtenues sont respectivement de $+0,23(P>0,05)$ ef $-0,59$ $(P<0,05)$.

\section{Discussion.}

Les résultats présentés dans cette étude montrent que chez les béliers de race lle-de-France, le pouvoir fécondant du sperme varie fortement selon la saison.

En mars ef en avril, la température moyenne n'affectant pas la reproduction des ovins sous nos latitudes, la lumière est seule à agir. La baisse de performances enregistrée à cette époque est donc bien causée par l'augmentation de la durée du jour. Ces résultałs confirment les données obtenues par Alberio (1976), Schanbacher (1979) et Colas (1979) sur des béliers soumis à un éclairement artificiel.

Salamon et Robinson (1962) et Fowler (1965) avaient déjà montré qu'après insémination, la fertilité naturelle des brebis Mérinos est plus élevée en automne qu'au 
printemps. Colas ef Brice (1976) ont obtenu des résultats similaires avec de la semence congelée sur des femelles traitées par des progestagènes, mais aucune de ces études $n$ 'avait $m$ is en évidence un écart aussi important. Cela tient sans doute au fait que les races ovines ne sont pas toutes aussi sensibles au photopériodisme (Smith ef Gordon, 1967 ; Islam et Land, 1977 ; Barrell et Lapwood, 1978). Par ailleurs, les éjaculats sont toujours triés dès la récolte, ce qui limite beaucoup les variations (alors que dans nos essais tous les prélèvements de semence ont été utilisés). Enfin, ces expériences n'ont pas eu lieu comme la nôtre aux époques où les différences de qualité du sperme sont maxima.

On peut penser que les fluctuations saisonnières chez le mâle n'apparaissent pas à l'état pur dans notre comparaison, puisque l'activité sexuelle de la femelle est elle aussi susceptible de varier. Cependant, il est maintenant établi qu'un traitement hormonal bien dosé permet d'obtenir une bonne synchronisation en période d'anœstrus, si les animaux sont correctement nourris et si l'intervalle part-insémination est suffisamment long (Cognié et al., 1975). De plus, il a été montré qu'on peut atteindre au printemps des taux de mise-bas comparables à ceux de l'automne, lorsque l'on fait appel à des béliers soumis à un éclairement décroissant (Colas et Guérin, 1979).

Si l'augmentation de la durée du jour agit de façon très marquée sur la fécondance du sperme, son effet n'a pas la même intensité chez tous les animaux. Les uns perdent en effet une grande partie de leurs aptitudes reproductrices ( $\sigma \mathrm{D}$ ef $\mathrm{E}$ ) alors que d'autres ( ${ }^{B} B$ et $C$ ) paraissent peu affectés par la saison. En automne, ces différences s'estompent, les béliers tendant à se comporter de façon beaucoup plus homogène.

De telles différences individuelles sonf un élément important à considérer dans la pratique. On a en effet souvent tendance à minimiser l'incidence du mâle dans la reproduction. Or, nous voyons qu'au printemps, après synchronisation des chaleurs, le taux de fertilité dépend dans une large mesure des béliers utilisés. Il est donc nécessaire de procéder à un tri sévère des animaux avant leur mise en service. Pour être efficace, ce choix ne peut s'opérer qu'au moment ou la variabilité entre géniteurs est maximum, c'est-à-dire en mars-avril, au moins pour la race lle-de-France.

Au printemps, il existe une forte corrélation négative entre la fécondance du sperme et son profil morphologique. Cette corrélation a été calculée sur un nombre restreint d'échantillons $(n=12)$, mais d'autres essais (non rapportés ici) ont confirmé la validité de ce contrôle que nous utilisons maintenant de façon courante au laboratoire. En d'autres termes, la numération des anomalies cellulaires donne une idée assez précise de la qualité de l'éjaculat chez le bélier dès la récolte. Ainsi, dans la pratique, il est conseillé d'éliminer les échantillons présentant plus de 20 à 25 p. 100 de malformations, puisque leur fécondance ne devrait guère dépasser 50 p. 100 (fig. 1).

L'élimination des mauvais géniteurs peut être réalisée à partir de cet examen. Nous savons en effet qu'au printemps, un animal conserve à peu près le même profil morphologique d'une année sur l'autre (Colas, 1980). Les sujets les plus sensibles à la lumière croissante auront donc toujours en mars-avril un pourcentage élevé d'anomalies et seront moins féconds. Il doit alors être possible de les détecter par la lecture de quelques frottis. 
Il est surprenant de constater que la liaison morphologie-fécondance, si étroiłe au printemps, esi quasi nulle en automne. Cela s'explique par le fait qu'en septembreoctobre, on ne rencontre guère que des anomalies légères de la pièce intermédiaire ou principale, dont la présence ne modifie pas de façon sensible les taux de réussite. Celles-ci n'ont donc rien à voir avec les malformations multiples (anomalies de la tête ou de l'acrosome, spermatozoïdes décapités, flagelles brisés, etc...) observées 6 mois plus tôt. Dès lors, il n'est pas anormal de ne plus trouver de différence significative de fertilité entre animaux.

Il aurait été intéressant de pouvoir classer au printemps les différents types d'anomalies d'après leur effet sur la fécondance. En dehors des travaux réalisés chez l'homme (David ef al., 1972 ; Sauvalle ef al., 1976), il n'existe, dans la littérature, aucune information dans ce domaine. Le peu d'échantillons dont nous disposions dans nos expériences ne nous a pas permis d'établir un tel ordre de priorité. Nous savons seulement que les malformations de la tête sont impliquées dans la baisse de qualité de l'éjaculat $(\S 2 c)$. Les défauts du flagelle, par contre, n'ont aucune influence, ce qui est difficile à admettre. En effet, si on conçoit volontiers que de légères courbures (automne) n'aient aucune incidence sur la fertilité, on comprend mal qu'il en soif de même pour des flagelles brisés, repliés ou anormalement courts. Une analyse plus fine de ces données nous aurait sans doute amené à nuancer notre résultat.

Contrairement à ce que l'on admet généralement chez les ovins, nous ne trouvons pas de relation entre la motilité d'un éjaculat et son pouvoir fécondant. Cela s'explique d'abord par le fait que la photopériode n'exerce aucun effet sur la turbulence du sperme (Colas, 1980). Par ailleurs, nous savons que la mesure de la motilité, telle qu'elle est pratiquée habituellement, est un critère subjectif. Enfin, les valeurs que nous avons enregistrées n'ont jamais été inférieures à 3 (note maximum 5). Pour des notes plus basses, il est probable que le taux de fertilité diminuerait car, dans ce cas, la proportion de cellules vivantes (qui est corrélée à la motilité selon Hafez, Badreldin ef Darwish, 1955) n'est plus suffisante pour permettre une fécondation normale. A l'intérieur des limites que nous avons rencontrées, l'examen de la motilité n'est donc pas un test sélectif. En saison sexuelle, lorsque les éjaculats ont une morphologie normale, il peut permettre d'éliminer les mauvais échantillons. En période de faible activité (printemps), son imprécision est telle qu'il devient difficile de l'utiliser.

Le pourcentage de spermatozoïdes morts ne subit de variations significatives qu'au cours de l'année 1978. A partir de là, on ne peut donc pas conclure que le sperme de bélier contient plus de cellules vivantes au printemps qu'en automne. Par ailleurs, une étude de l'évolution hebdomadaire de ce paramètre, réalisée pendant deux périodes consécutives de 12 mois sur les mêmes animaux (Colas, non publié), nous a permis de montrer qu'il n'est pas soumis à l'influence de la lumière naturelle. Le fait de ne pas trouver de relation entre pourcentages de cellules colorées et fertilité ne doit donc pas surprendre. II montre seulement que les doses de semence que nous avons utilisées devaient contenir un nombre suffisant de gamétes vivants et que des spermatozoïdes colorés ne sont pas pour autant fécondants. Ces résultats ne confirment pas les données de Hulet et Ercanbrack (1962) et de Hulet, Foote et Blackwell (1965), obtenues dans des conditions fort éloignées des nôtres (accouplement, saison sexuelle, prélèvement du sperme dans les voies génitales). Ils indiquent néanmoins qu'on ne peut se fier au test de coloration pour apprécier la qualité d'un éjaculat. 


\section{Conclusion.}

Chez le bélier adulte lle-de-France, le pouvoir fécondant du sperme est significativement plus faible au printemps (mars-avril) qu'en automne (septembre). Il existe des variations individuelles, mais celles-ci ne sont importantes et significatives qu'au printemps (mars-avril).

A cette époque, il existe une relation étroite et inverse entre la fécondance de l'éjaculat et la proportion de spermatozoïdes anormaux. En automne (septembre) où les malformations sont beaucoup moins nombreuses et de moindre importance, cette relation disparaît. On peut donc apprécier la qualité de la semence chez le bélier par un contrôle de ses anomalies.

De tels écarts individuels permettent d'envisager le tri des animaux les moins aptes à se reproduire au printemps. Ce choix doit être réalisé en mars-avril par le test morphologique.

La motilité massale ef le pourcentage de spermatozoìdes colorés de l'éjaculat ne sont liés à sa fécondance, ni au printemps ni à l'automne.

Reçu en septembre 1980.

Accepté en novembre 1980.

Remerciements. - Nous tenons à exprimer nos remerciements à $M$. Courot pour les commentaires qu'il a faits sur cette étude, et au personnel de la bergerie qui a assuré les inséminations artificielles.

\section{Références}

ALBERIO R., 1976. Rôle de la photopériode dans le développement de la fonction de reproduction chez l'agneau lle-de-France, de la naissance d 21 mois. Thèse Doct. 3e Cycle, Univ. Paris VI, 57 pp.

BARRELL G. K., LAPWOOD K. R., 1978. Seasonality of semen production and plasma luteinizing hormone, testosterone and prolactin levels in Romney, Merino and Polled Dorset rams. Anim. Reprod. Sci., 1, 213-228.

COGNIE Y., HERNANDEZ-BARRETO M., SAUMANDE J., 1975. Low fertility in nursing ewes during the non-breeding season. Ann. Biol. onim. Bioch. Biophys., 15, 329-343.

COLAS G., 1975. The use of progestagen SC 9980 as an aid for artificial insemination in ewes. Ann. Biol. anim. Bioch. Biophys., 15, 317-327.

COLAS G., 1979. Fertility in the ewe after artificial insemination with fresh and frozen semen at the induced oestrus and influence of the photoperiod on the semen quality of the ram. Livesiock Prod. Sci., 6, 153-166.

COLAS G.,1980. Variations saisonnières de la qualité du sperme chez le bélier fle-de-France. I. Etude de la morphologie cellulaire et de la motilité massale. Nutrition, Reprod. Dévelop., 20, 1789-1799.

COLAS G., BRICE G., 1976. Seasonal variations of the fertilizing capacity of the deep-frozen ram semen. VIIlih Congr. onim. Reprod. artif. Insem., Krakow, IV, 977-980.

COLAS G., GUÉRIN Y., 1979. L'insémination artificielle chez les ovins : acquisitions et perspectives. $5^{e}$ journées Rech. Ov., Copr. INRA-ITOVIC, 162-185.

DAVID G., BISSON J. P., JOUANNET P., CZYGLIK F., GERNIGON C., ALEXANDRE C., DREYFUS G., 1972. Les tératospermies, 81-102. In Fécondité et stérilité du mâle. Acquisitions récentes. Masson éd., Paris.

FOWLER D. G., 1965. Semen quality of Merino rams. 2. The effects of seasonal changes in daylength on semen quality. Aust. J. exp. Agric. anim. Husb., 5, 247-251. 
HAFEZ E. S. E., BADRELDIN A. L., DARWISH Y. H., 1955. Seasonal variations in semen characteristics of sheep in the subtropics. J. agric. Sci., 45, 283-292.

HULET C. V., ERCANBRACK S. K., 1962. A fertility index for rams. J. anim. Sci., 21, 489-493.

HULET C. V., FOOTE W. C., BLACKWELL R. L., 1965. Relationship of semen quality and fertility in the ram to fecundity in the ewe. J. Reprod. Fert., 9, 311-315.

ISLAM A. B. M. M., LAND R. B., 1977 . Seasonal variation in testis diameter and sperm output of rams of breeds of different prolificacy. Anim. Prod., 25, 311-317.

SALAMON S., ROBINSON T. J., 1962. Studies on the artificial insemination of merino sheep. I. The effects of frequency of season of insemination, age of the ewe, rams and milk diluents on lambing performance. Aust. J. agric. Res., 13, 52-68.

SAUVALLE A., HERLICOVIEZ D., DESHAYES M. J., TZARD J., 1976. Analyse de 144 spermocytogrammes. Arch. Anat. cytol. path., 24, 369-377.

SCHANBACHER B. D., 1979. Increased lamb production with rams exposed to short daylengths during the non-breeding season. J. anim. Sci., 49, 927-932.

SMITH P., GORDON I., 1967. Seasonal and breed variations in the semen characteristics of rams in Ireland. Irish Vet. J., 21, 222-233. 